\title{
A case of fatal haemorrhage due to ruptured varicose veins
}

\author{
Rathnaweera RHAI \\ Department of Forensic Medicine, Faculty of Medicine, University of Ruhuna, Galle, Sri Lanka.
}

Correspondence: Dr.RHAI Rathnaweera

e-mail: ajithrathnaweera@gmail.com

D https://orcid.org/0000-0002-7418-2981

\section{Introduction}

Presence of varicose veins in lower limbs is a common condition in the community affecting 14 to $59 \%$ of the population (1). It has been estimated that $>20$ million people in the United States have some form of symptomatic chronic varicose vein- related conditions (2). Varicose veins appear as tortuous dilated veins with associated oedema and induration of the skin (1). The most frequently affected veins are the superficial veins of the lower extremities (3). While complications such as leg oedema, varicose eczema and ulceration are not unusual, fatal haemorrhage from rupture is a rare event in most forensic practices. This rupture can happen spontaneously or as a result of minor trauma and in general is not fatal (2). However, if unattended, a fatal outcome can occur in a small percentage of cases (3). The investigation of such cases is usually straightforward, although the amount of blood at the scene, and the elderly and frail nature of many of the victims may raise the possibility of an assault.

\section{Case presentation}

A 60-year-old woman was found dead in bed at her home by a neighbour. As there was blood pooling all over the place, the neighbor had immediately contacted the police. According to the neighbour, the deceased was living alone at this place for the last six months after the demise of her husband. She was suffering from diabetes mellitus and hypertension, however was not on regular treatment. She also had varicose veins over her lower limbs, for which she occasionally took treatment from a general practitioner. She was not on any anticoagulants at the moment.
A scene visit was arranged by the police with the participation of the magistrate, scene of crime officers and the judicial medical officer. There were no signs of breaking and entering at the scene. The deceased was found lying on the bed with blood staining over her left shin and foot. Pooling of blood was seen at the foot end of the bed (Figure 1), the bed room floor and the bathroom floor. A pattern of fine elliptical bloodstains around blood pools were present on the floor (Figure 2).

Autopsy was performed after the body was transported to the mortuary. The body was that of an elderly female with features in keeping with the mentioned age. No fresh or old injuries were found over the body suggestive of assault. Evidence of chronic varicose veins was present over both lower limbs. Marked discolouration was seen over the medial aspect of the left lower leg and ankle. An acute varicose ulcer measuring $2 \times 3$ millimeters in size was present anterior to the medial malleolus of the left lower leg (Figure 3). Associated haemorrhage was seen in and around the wound. The ulcer was shallow in depth with irregular shaped edges. There was oedema and varicose eczema surrounding the ulcer. The systematic dissection of the ulcer revealed communication with a superficial varicosity that drained into the great saphenous vein. The only other significant finding was the marked coronary artery atherosclerosis. The left main, left circumflex and right coronary artery showed significant atherosclerotic narrowing. Toxicological evaluation was negative for common poisons and illicit drugs. 


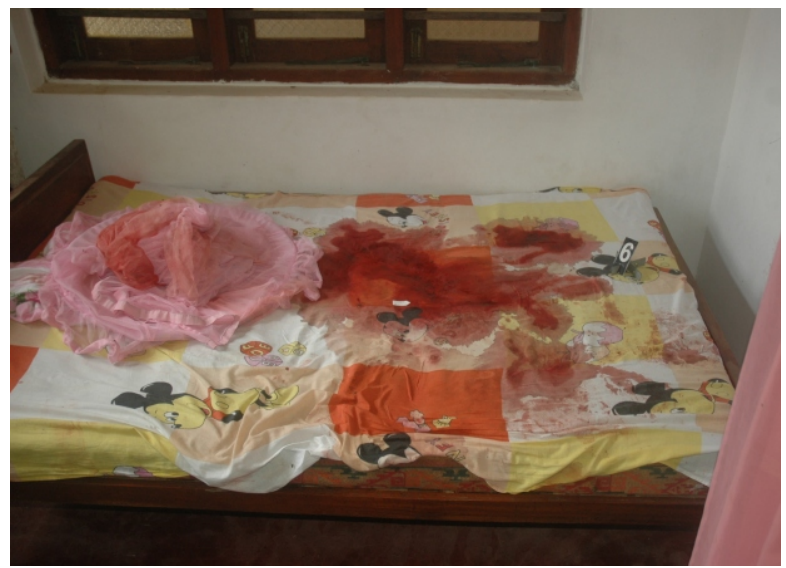

Figure 1: Pooling of blood on the foot end of the bed

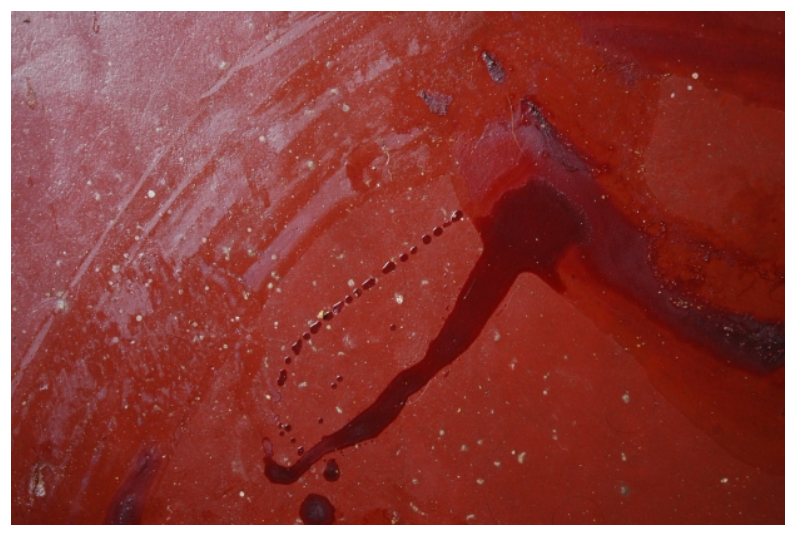

Figure 2: Fine elliptical bloodstain pattern around blood pools on the floor

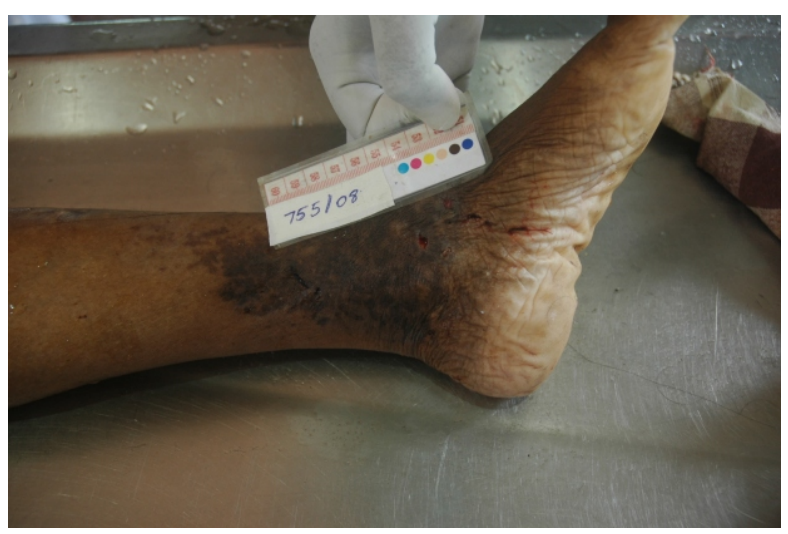

Figure 3: Acute varicose ulcer with discolouration of the skin over the medial malleolus

\section{Discussion}

Varicose veins are dilated and tortuous veins that are usually found in the legs, associated with valvular incompetence. The aetiology is multifactorial, with suggestions that congenitally defective valves may be responsible for chronic venous distension, being superseded by the theory that valve incompetence follows dilatation of inherently weak vein walls (4).

Varicose veins are extremely common in the adult population, occurring in $10 \%$ to $40 \%$ of western men and $25 \%$ to $33 \%$ of western women, with the most commonly affected vein being the great saphenous (5).

The reported cases illustrate a number of characteristic features of deaths due to haemorrhage from varicose veins. Victims are characteristically elderly, and haemorrhage may occur from rupture of an intact varix, sometimes associated with minor trauma, from chronic venous ulceration into a superficial varix, or from more substantial trauma, causing laceration of skin and soft tissues overlying a varicosity. The fragility of skin and soft tissues in the elderly means that more significant injuries may result from relatively less severe trauma than in younger individuals (6).

Failure to adopt swift measures to control bleeding is a characteristic feature in these cases. This failure to control the bleeding by way of using simple measures, such as applying direct pressure or by elevating the affected limb, could be due to many reasons. Lack of awareness and failure to recognise the seriousness of bleeding and absence of training in simple first aid measures are commonly known causes. Underlying psychiatric conditions such as dementia, which can alter the level of mental state and memory and use of some of the medications and substance of abuse also could play a vital role in this scenario.

Co-morbidities such as ischaemic heart disease may predispose to death following significant exsanguination, or may result in death from less severe blood loss than in an individual with unimpaired coronary artery circulation. Alcohol may enhance the speed of bleeding due to peripheral vasodilation, and anticoagulant medications, may also predispose to significant haemorrhage from apparently trivial defects (7).

Bloodstain pattern analysis and interpretation are a standard part of many crime scene investigations. Examination of the characteristics of bloodstains may provide important information about the events leading up to their deposition. 
Classically, arterial bleeding is associated with a projected bloodstain pattern with significant volume and spines (the pointed edges that radiate out from the center of stains due to the volume and force of the haemorrhage) (8). Clusters of large elliptical stains and drip patterns occur with significant lacerations or incisions of large arteries causing an arterial spurt or gush that differs from impact spatters or low-velocity, free-falling droplets of blood (5). Lesser injuries to smaller arteries may produce a much finer pattern from smaller droplets. As blood that escapes from veins is under much lower pressure it tends to pool until gravitational forces acting on it exceed the surface tension of the blood. This result in the formation of spherical droplets in free-fall that produce approximately circular stains upon impact with a horizontal surface such as a floor. These stains are characterized by their large size $(13-21.5 \mathrm{~mm})$ and their lack of directional indicators (5).

The unique situation of lower limb varicose veins, with incompetent valves and pooled venous blood under higher than normal pressure, may result in fine 'arterial-type' blood spatter, as in this case, if a small defect occurs in the wall of a varicosity. An awareness of this possibility may assist in the evaluation of death scenes where there is extensive haemorrhage with apparent arterial blood spray. The identification of an acutely ulcerated lower leg varicosity beneath adherent blood clot, with blood spatter either on the floor, or at a level that corresponds to the height above the floor of the vascular defect, are all features that support higher than normal pressure venous, and not arterial, haemorrhage.

\section{Conclusions}

This case has demonstrated the uncommon occurrence of death due to haemorrhage from varicose veins. Despite the rarity of these events, the entirely preventable nature of the deaths by the application of simple first aid procedures suggests that the possibility of this outcome should be discussed with all individuals who are afflicted with varicose veins. The speed at which death may occur under these circumstances, underlines the vulnerability of socially isolated individuals to a lethal outcome once a varicosity has been breached.
Autopsy examinations should include careful layer dissection of the area of haemorrhage to demonstrate ruptured varices and to enable directed histologic examination of ulcers. Toxicological evaluation and drug histories may provide useful information on drugs or medications that may enhance bleeding. Full autopsy evaluation is also required to determine whether there are underlying organic diseases, such as ischaemic heart disease, that may have contributed to or hastened death or whether there is evidence of any sequelae to collapse from blood loss, such as hypothermia, that may also have played a role in the terminal mechanism.

\section{References}

1. Lane RJ, Cuzzilla ML. Aetiology of varicose veins: haemodynamics. ANZJ Surg, 2003; 73: 874-876.

2. Golledge J, Quigley FG. Pathogenesis of varicose veins. Eur J Vasc Endovasc Surg, 2003; 25: 319-324.

3. Pistorius MA. Chronic venous insufficiency: The genetic influence. Angiol Suppl, 2003; 54: S5-12.

4. Hingorani A, Chait J, Kibrik $\mathrm{P}$, et al. Spontaneous haemorrhage from varicose veins: A single-center experience. Venous and Lymphatic Disorders. Journal of Vascular Surgery, 2020; 8(1): 106-109.

5. Crawford JM, Lal KB, Duran W, et al. Pathophysiology of venous ulceration. Venous and Lymphatic Disorders. Journal of Vascular Surgery, 2017; 5(4): 596-605.

6. Fragkouli K, Mitselou A, Boumba V, et al. Unusual death due to a bleeding from a varicose vein: a case report. BMC Research Notes. 2012; 5: 488.

7. Aquila I, Sacco M, Gratteri S, et al. Sudden death by rupture of a varicose vein: Case report and review of literature. Medico-Legal Journal, 2017; 85(1): 47-50.

8. Jelev L, Alexandrov A. A case of fatal bleeding from acute varicose leg ulcer: Clinico-pathological characteristics. EJVES Extra, 2011;21: e33-e35. 